\title{
A rare manifestation of extrapulmonary tuberculosis: left ventricular cardiac tuberculoma in an HIV infected male "case report"
}

\author{
Shokoufeh Hajsadeghi ${ }^{1}$, Aida Iranpour ${ }^{1}$, Saeed Kalantari ${ }^{2}$, Farzad Dashti ${ }^{1}$ \\ ${ }^{1}$ Research Center for Prevention of Cardiovascular Disease, Institute of Endocrinology \& Metabolism, ${ }^{2}$ Research Center for Antimicrobial \\ Resistance, Iran University of Medical Sciences, Tehran, Iran \\ Correspondence to: Farzad Dashti, MD. Research Center for Prevention of Cardiovascular Disease, Institute of Endocrinology \& Metabolism, Iran \\ University of Medical Sciences, Tehran, Iran. Email: Farzad.d@gmail.com.
}

\begin{abstract}
Cardiac tuberculosis (TB) is rare and most commonly manifests itself as tuberculous pericarditis. Involvement of other parts of the heart is unusual and descriptions in the literature are confined to case reports regarding mainly pericardial TB and very few cases of cardiac tuberculoma. Tuberculomas are space occupying lesions most commonly found in the brain of immunocompromised individuals. These space occupying lesions previously described only after autopsies are now more diagnosed with the use of advanced imaging techniques. Herein, we describe a first case of pericardial TB manifesting as left ventricular (LV) cardiac tuberculoma in a 34-year-old human immunodeficiency virus (HIV) and hepatitis C virus (HCV) infected male. Upon presentation the patient complained mainly of progressive dyspnoea over the past month. Primary investigations including chest computed tomography (CT) scan and transthoracic echocardiography (TTE) suggested probable diagnosis of cardiac and pericardial TB which was later confirmed by histopathological modalities. The patient received anti-TB therapy along with surgical subtotal pericardiotomy which resulted in improvement of symptoms, complete resolution of the mass and reduction in the size of pericardial thickening. Although very rare it is crucial to bear in mind the importance of having cardiac tuberculoma as differential diagnosis in patients with a cardiac mass and implement the optimum diagnostic and therapeutic courses.
\end{abstract}

Keywords: Tuberculoma; constrictive pericarditis; human immunodeficiency virus (HIV); case report

Submitted Apr 30, 2020. Accepted for publication Aug 05, 2020.

doi: $10.21037 / \mathrm{cdt}-20-446$

View this article at: http://dx.doi.org/10.21037/cdt-20-446

\section{Introduction}

Pericarditis is a very rare manifestation of extrapulmonary tuberculosis $(\mathrm{TB})$ that occurs in approximately $1 \%$ to $2 \%$ of patients with pulmonary TB (1). In developing countries with a high prevalence of human immunodeficiency virus (HIV), there has been a dramatic increase in all forms of extrapulmonary TB particularly tuberculous pericarditis (1). Myocardial tuberculoma occurrence is extremely rare and only very few cases have been reported (2). Due to rarity of the disease and scarcity of informative studies, the optimal diagnostic workup is not common knowledge, and the appropriate management has not been well defined (3). Here we present a case to highlight the diagnostic work up and management of cardiac tuberculoma in addition to pericardial TB. We present the following article in accordance with the CARE reporting checklist (available at http://dx.doi.org/10.21037/cdt-20-446).

\section{Case presentation}

A 37-year-old, opium addict, male presented to emergency department with complaint of progressive dyspnoea. His dyspnoea was at functional class (FC) II and had increased to FC IV over the last 20 days. He also complained of night sweets, chills and a $10 \mathrm{~kg}$ weight loss over the last month. Past medical history was remarkable for HIV 

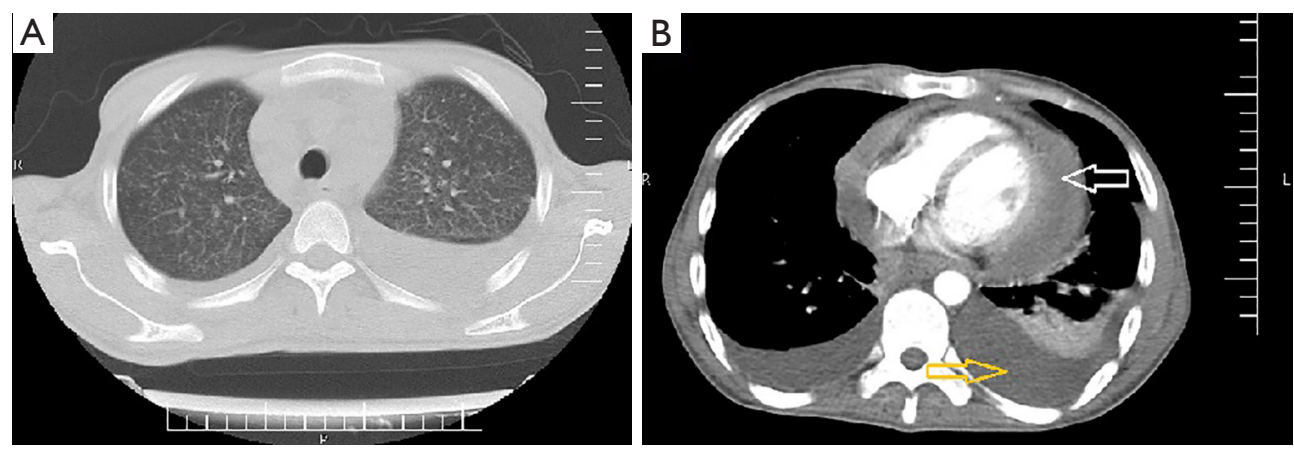

Figure 1 Chest CT scan. (A) Axial lung window showed bilateral diffuse micro-nodular opacities suggestive of miliary TB; (B) axial mediastinal window showed bilateral pleural effusion (yellow arrow) associated with pericardial effusion (white arrow). CT, computed tomography; TB, tuberculosis.

and hepatitis $\mathrm{C}$ virus (HCV) infection. Upon physical examination lower extremity edema and increased jugular venous pressure (JVP) were noticed. He was started on antiretroviral therapy including tenofovir, emtricitabine and efavirance about 2 weeks before his symptoms commence. The patient was admitted and primary investigations including chest computed tomography (CT) scan revealed bilateral pleural effusion, miliary pattern in both lungs and pericardial thickening including pericardial effusion (Figure 1). Preliminary lab data were as follows; white blood cell (WBC): 2,500 with segment of $84.2 \%$ and lymphocytes of $7.6 \%$ (CD4 count was 64 ), hemoglobin $8.8 \mathrm{mg} / \mathrm{dL}$ and platelets $104,000 / \mu \mathrm{L}$. Based on imaging studies a preliminary diagnosis of $\mathrm{TB}$ was proposed and standard anti-TB medication and $50 \mathrm{mg}$ prednisolone daily was started. To further elucidate the cause of pericardial effusion and thickening transthoracic echocardiography (TTE) was performed which showed significant thickening of pericardium [24 $\mathrm{mm}$ posterior to left ventricular (LV)] with mild pericardial effusion [6 $\mathrm{mm}$ anterior to right ventricular (RV)] with constrictive physiology (Figure 2). Two weeks after treatment, once more we performed TTE that revealed significant thickening of intra-pericardial space (21 $\mathrm{mm}$ posterolateral to $\mathrm{LV}$ ) with constrictive physiology [ $40 \%$ inspiratory variation in mitral valve (MV) inflow with septal bounce and E: $17 \mathrm{~cm} / \mathrm{sec}$ in septal wall comparison with $8 \mathrm{~cm} / \mathrm{sec}$ in lateral wall compatible with annulus reversus]. Also, a moderate size $(6 \times 8 \mathrm{~mm})$ pedunculated mobile mass at $L V$ apex suggestive for $L V$ thrombus butar are diagnosis granuloma (tuberculoma) was considered due to history of TB (Figure 2). The patient anti-TB treatment was continued and due to inadequate response to therapy surgery was considered. Subsequently the patient underwent sternotomy which revealed adherence of pericardium into epicardium and myocardium and caseafied changes throughout the heart particularly at the left side. Accordingly, subtotal pericardiotomy was performed and histopathological examination revealed granulomatous inflammation including epithelioid cell granuloma, multinucleated giant cells and small necrotic areas with no signs of malignancy which later confirmed caseous granuloma (Figure 3).

The anti-TB treatment was continued. Upon follow ups with serial echocardiography the tuberculoma was resolved and pericardial thickening reduced in size but was still persistent.

All procedures performed in studies involving human participants were in accordance with the ethical standards of the institutional and/or national research committee(s) and with the Helsinki Declaration (as revised in 2013). Written informed consent was obtained from the patient for publication of this study and any accompanying images.

\section{Discussion}

Cardiac TB is a rare disease and it usually represents as tuberculous pericarditis which can result in constrictive pericarditis (4). Involvement of other parts of the heart is unusual and only very few cases have reported involvement of myocardium and endocardium (4). On the basis of histopathological appearance myocardial TB occurs as three different forms including miliary form, nodular form and tuberculoma (5). Tuberculoma, a tumor-like manifestation of the disease, was first reported by Morgagni in 1761 (5). Single or multiple cardiac tuberculomas are extremely rare and most commonly are observed in the right heart chambers, especially in right atrium wall (6). Tuberculomas are often well circumscribed and sharply demarcated from the surrounding 

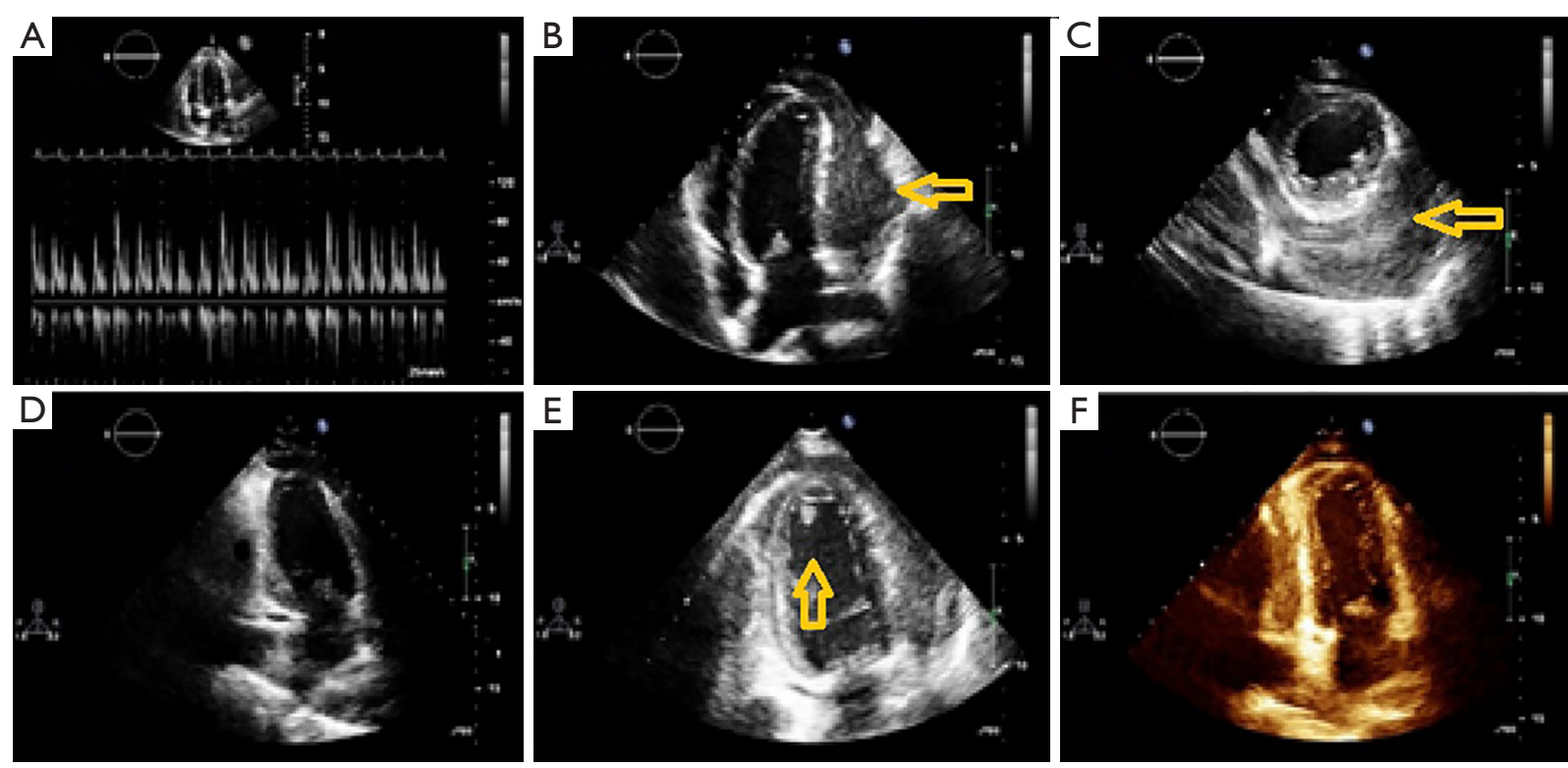

Figure 2 TTE images of the patient before, in the middle and after treatment. (A) (Inspiratory view), (B) (four chamber view) and (C) (two chamber view) show the pericardial thickness (yellow arrow); (D) shows four chamber view before cardiac tuberculoma appears; (E) displays the tuberculoma at the apex of LV (yellow arrow) and (F) shows disappearance of the tuberculoma after treatment. TTE, transthoracic echocardiography; LV, left ventricular.
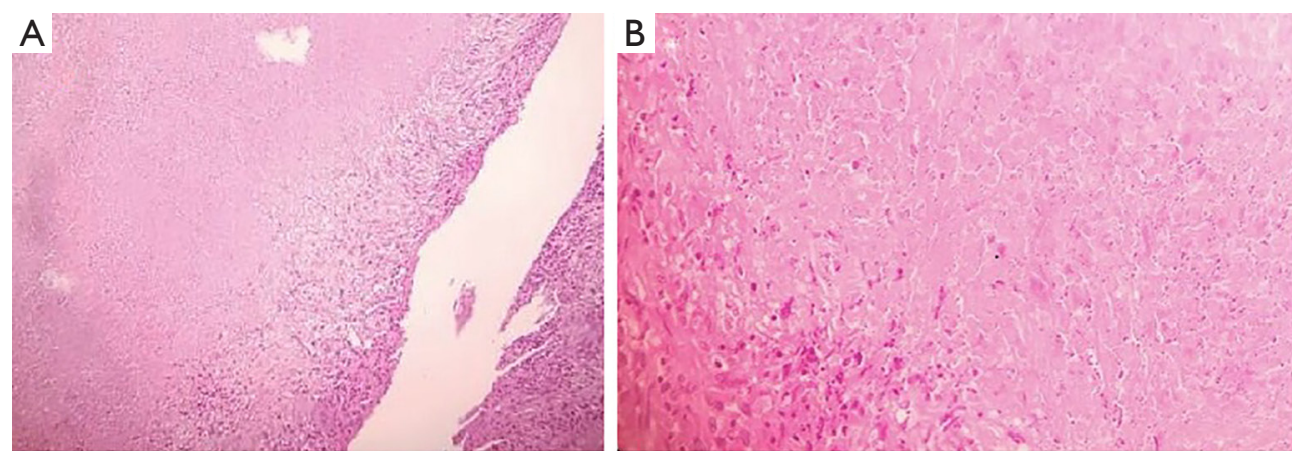

Figure 3 Aggregation of lymphohistiocytes forming caseating granulomatose inflammation. (A) Caseous necrosis surrounded by epithelioid histiocytes (H\&E, $\times 40)$; (B) higher view shows large area of caseous necrosis next to aggregates of epithelioid histiocytes, intermingled with scattered lymphocytes $(\mathrm{H} \& \mathrm{E}, \times 100)$.

tissues (6). They can occur as the only manifestation of active $\mathrm{TB}$ or can be associated with miliary $\mathrm{TB}(5)$.

To our knowledge, this is the first report of a cardiac tuberculoma representing as a large size pedunculated mobile mass at LV apex. This case showed an unusual cardiac complication of TB not only causing pericardial thickening as well as pericardial effusion but also involving the LV by means of a large size cardiac tuberculoma. In our case 2 weeks before the beginning of his symptoms he was started on antiretroviral therapy, this can suggest the possibility of hypothesis that the exacerbations in symptoms and signs, laboratory and radiographic manifestations in this patient occurred through the mechanism termed the Immune Reconstitution Inflammatory Syndrome (IRIS) which has been associated with administration of antiretroviral regimens (7). IRIS is particularly common among patients who are HIV infected and immunocompromised with extrapulmonary TB (7). This reaction is thought to be associated with improved immune function elicited by antigens released as the 
bacilli are killed. In other words, unmasking IRIS is an antiretroviral associated inflammatory manifestation of a subclinical infection with a hastened presentation. In this latter form signs and symptoms not clinically apparent before, appear during antiretroviral therapy (7). One of the proposed treatments for this phenomenon is the use of glucocorticoids that we also used in our patient (7).

Imaging techniques particularly echocardiography are the cornerstone of characterizing intra-cardiac masses. Nevertheless, confirmation of the diagnosis is achieved via biopsy of the mass and its histological features in addition to the gold standard method of mycobacterial culture.

The treatment of choice seems to be anti-TB medication with duration of approximately 9 months. Previous cases have reported definitive cure in terms of complete recovery of patient's signs and symptoms along with imaging evidence of cardiac involvement resolution without surgery but in our patient this was not the case even with the help of surgical therapy (5). Indications for surgical management include uncertain diagnosis, threatening thromboembolism, refractory malignant arrhythmias, hemodynamically significant obstruction and inadequate response to medical therapy (5).

In conclusion, this case revealed a peculiar cardiac complication of $\mathrm{TB}$ which is exceedingly rare and apart from very few cases reported so far others were not diagnosed while the patient was alive. Advanced imaging modalities now have given us the capability of earlier diagnosis and surveillance. Cardiac tuberculoma may regress after anti-TB therapy, and surgery may be needed to help with the diagnosis as well as the therapy.

\section{Acknowledgments}

Funding: None.

\section{Footnote}

Reporting Checklist: The authors present the study in accordance with the CARE reporting checklist. Available at http://dx.doi.org/10.21037/cdt-20-446

Conflicts of Interest: All authors have completed the ICMJE uniform disclosure form (available at http://dx.doi. org/10.21037/cdt-20-446). The authors have no conflicts of interest to declare.

Ethical Statement: The authors are accountable for all aspects of the work in ensuring that questions related to the accuracy or integrity of any part of the work are appropriately investigated and resolved. All procedures performed in studies involving human participants were in accordance with the ethical standards of the institutional and/or national research committee(s) and with the Helsinki Declaration (as revised in 2013). Written informed consent was obtained from the patient for publication of this study and any accompanying images.

Open Access Statement: This is an Open Access article distributed in accordance with the Creative Commons Attribution-NonCommercial-NoDerivs 4.0 International License (CC BY-NC-ND 4.0), which permits the noncommercial replication and distribution of the article with the strict proviso that no changes or edits are made and the original work is properly cited (including links to both the formal publication through the relevant DOI and the license). See: https://creativecommons.org/licenses/by-nc-nd/4.0/.

\section{References}

1. Mayosi BM, Burgess LJ, Doubell AF. Tuberculous pericarditis. Circulation 2005;112:3608-16.

2. Syed FF, Mayosi BM. A modern approach to tuberculous pericarditis. Prog Cardiovasc Dis 2007;50:218-36.

3. Trautner BW, Darouiche RO. Tuberculous pericarditis: optimal diagnosis and management. Clin Infect Dis 2001;33:954-61.

4. Al-Nasser I, Anwar AM, Nosir YF, et al. Bicaval obstruction complicating right atrial tuberculoma: the diagnostic value of cardiovascular MR. J Cardiovasc Magn Reson 2008;10:60.

5. Säll O, Cha SO, Holmberg H. Diagnostic challenges in a patient with myocardial tuberculoma: a case report. Int J Surg Case Rep 2016;29:201-3.

6. Cantinotti $M$, De Gaudio M, de Martino M, et al. Intracardiac left atrial tuberculoma in an eleven-monthold infant: case report. BMC Infect Dis 2011;11:359.

7. Lanzafame M, Vento S. Tuberculosis-immune reconstitution inflammatory syndrome. J Clin Tuberc Other Mycobact Dis 2016;3:6-9.

Cite this article as: Hajsadeghi S, Iranpour A, Kalantari S, Dashti F. A rare manifestation of extrapulmonary tuberculosis: left ventricular cardiac tuberculoma in an HIV infected male “case report". Cardiovasc Diagn Ther 2020;10(5):1341-1344. doi: $10.21037 / \mathrm{cdt}-20-446$ 